\title{
Electron Microscopy Investigation on Empty Germanium Clathrate-II
}

\author{
Reiner Ramlau*, Michael Baitinger ${ }^{*}$, Zhongjia Tang ${ }^{* *}$, Arnold M. Guloy ${ }^{* *}$, and Yuri Grin ${ }^{*}$ \\ * Max-Planck-Institut für Chemische Physik fester Stoffe, Nöthnitzer Str. 40, 01187 Dresden, \\ Germany \\ ** Department of Chemistry and Center for Materials Chemistry, University of Houston, Houston, \\ TX 77204-5003
}

The empty clathrate-II of germanium (Fig. 1) is an open, covalently bonded 3D network of tetrahedrally coordinated $\mathrm{Ge}$ atoms forming pentagon-dodecahedral $\mathrm{Ge}_{20}$ and hexakaidecahedral $\mathrm{Ge}_{28}$ cages. Each unit cell contains 16 pentagon dodecahedra and 8 hexakaidecahedra, which provide space for 24 cage positions in a completely filled clathrate. Consequently, the empty clathrate-II is best described by the formula $\square_{24} \mathrm{Ge}_{136}$.

Theoretical studies have predicted a relatively high stability of the clathrates-II $\mathrm{Na}_{x} \mathrm{Si}_{136}$ and $\mathrm{Na}_{x} \mathrm{Ge}_{136}(x \rightarrow 0)$ with respect to the diamond form of $\mathrm{Si}$ and Ge [1], and various methods for their synthesis have been suggested. In contrast to $\mathrm{Na}_{x} \mathrm{Si}_{136}(x<1)$, which can be synthesized as a mixture with silicon by thermal decomposition of $\mathrm{NaSi}$ in dynamic vacuum [2], all attempts to synthesize an empty or almost empty clathrate-II of germanium failed so far. Attempts to prepare guest-free Si and Ge clathrates using direct reactions have been unsuccessful in any case.

We could recently report the high-yield synthesis of empty clathrate $\square_{24} \mathrm{Ge}_{136}$ through soft oxidation of $\mathrm{Ge}_{9}{ }^{4-}$ Zintl anions [3]. X-ray powder diffraction patterns of the polycrystalline product indicated the presence of a small content of $\alpha-G e$. The major phase was completely indexed with the facecentered cubic lattice and a lattice parameter of $a=15.2152(1) \AA$. Full-profile refinement [4] of the crystal structure from powder diffraction data confirmed the clathrate-II germanium framework (space group $F d \overline{3} m$ ). Within the e.s.d. the cationic positions were found to be empty. Highresolution transmission electron microscopy (HRTEM) showed that the product contained one major crystalline phase $\left(\square_{24} \mathrm{Ge}_{136}\right)$ and two minor phases, one being amorphous and one crystalline. The selected-area electron diffraction (SAED) patterns confirmed the cubic symmetry and unit-cell parameters of the clathrate-II structure (Fig. 2). The agreement between experimental and simulated images (Fig. 3) was rather good, thus supporting the results of the X-ray crystal-structure refinement. However, the complete absence of sodium in the cages of the structure cannot doubtlessly be deduced from HRTEM. Only investigations by electron energy-loss spectroscopy (EELS) clearly revealed the absence of $\mathrm{Na}$ in the clathrate phase: no $\mathrm{Na}-K$ edge at $1072 \mathrm{eV}$ was observed in the EEL spectra (Fig. 4). Thus the cages of crystalline $\square_{24} \mathrm{Ge}_{136}$ are essentially empty.

\section{References}

[1] J. Dong and O. F.Sankey, J. Phys.: Condens. Matter 11 (1999) 6129.

[2] K. Ammar et al., Solid State Sciences 6 (2004) 393.

[3] A. M. Guloy, R. Ramlau, Z. Tang, M. Baitinger and Yu. Grin, submitted.

[4] L. G. Akselrud et al., Mater. Sci. Forum 133-136 (1993) 335. 


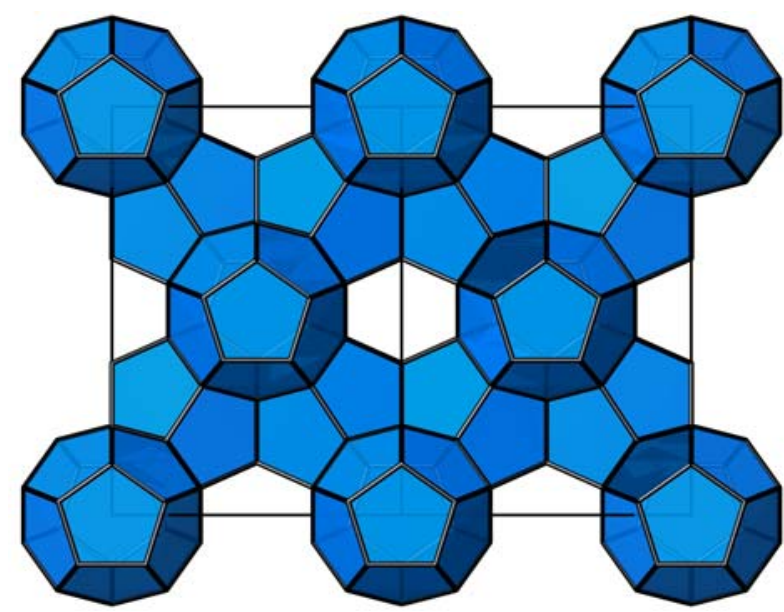

FIG. 1. Unit cell of clathrate-II $\square_{24} \mathrm{Ge}_{136}$ viewed along [110]. The crystal structure contains two basic motifs: distorted pentagon dodecahedra (blue) with $20 \mathrm{Ge}$ atoms and hexakaidecahedra (not highlighted) with 28 Ge atoms.

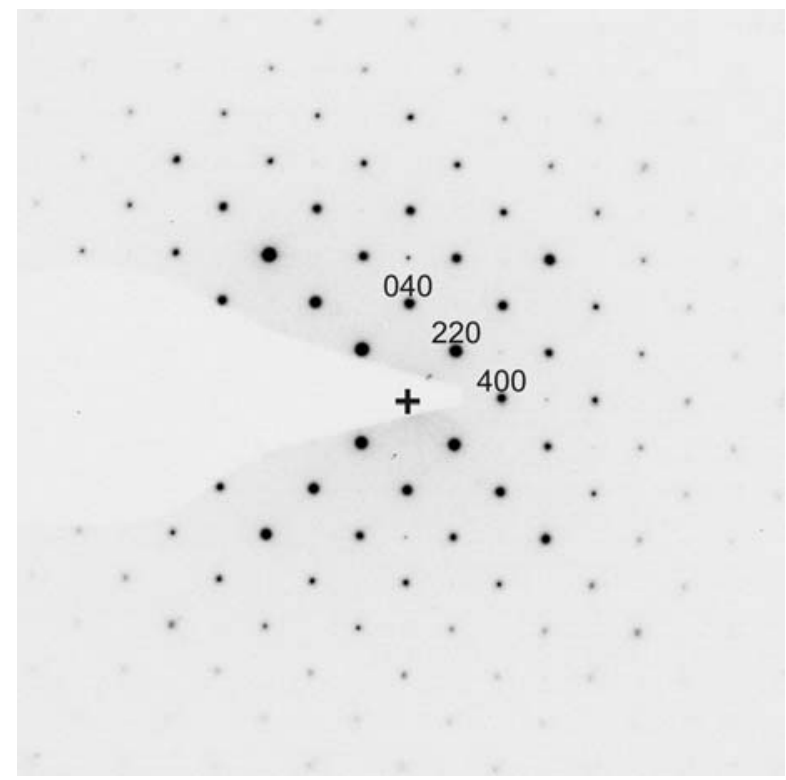

FIG. 2. SAED pattern of clathrate $\square_{24} \mathrm{Ge}_{136}$ for the [100] zone axis.
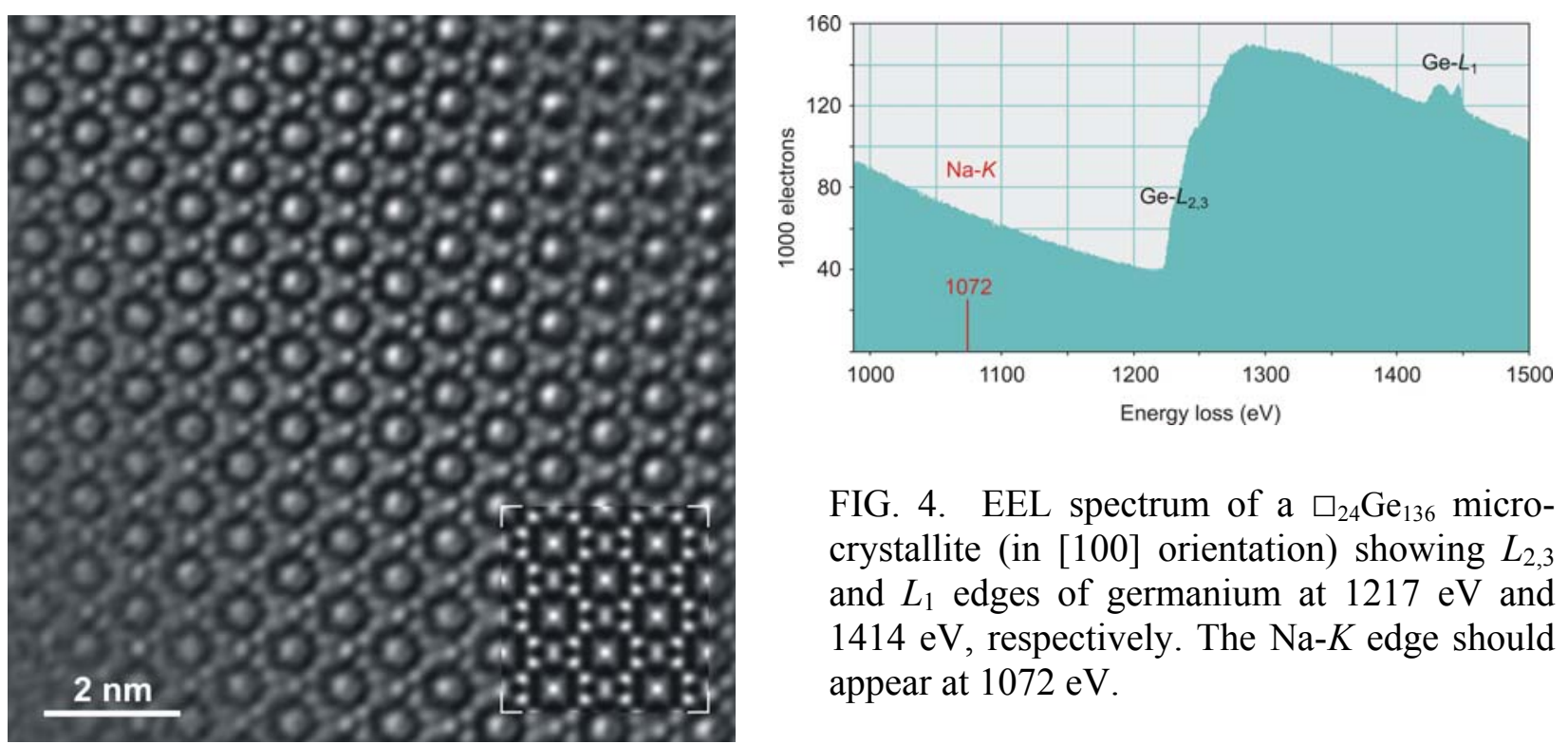

FIG. 4. EEL spectrum of a $\square_{24} \mathrm{Ge}_{136}$ microcrystallite (in [100] orientation) showing $L_{2,3}$ and $L_{1}$ edges of germanium at $1217 \mathrm{eV}$ and $1414 \mathrm{eV}$, respectively. The Na- $K$ edge should appear at $1072 \mathrm{eV}$.

FIG. 3. HRTEM image of clathrate $\square_{24} \mathrm{Ge}_{136}$ taken around SCHERZER optimum focus for zone axis [110]. A simulated image (multi-slice formalism) is inserted; defocus: $\Delta f=-50 \mathrm{~nm}$, specimen thickness: $t=4.3 \mathrm{~nm}$. The Tecnai G2-F30 electron microscope with super-twin lens $\left(C_{S}=1.2 \mathrm{~mm}\right)$ was operated at $300 \mathrm{kV}$ (point resolution $0.2 \mathrm{~nm}$ ). 